\title{
Simulation of Heterogeneous Network Vehicles
}

\author{
Suad Kasapovic ${ }^{1}$, Samra Mujacic ${ }^{1}$, Lejla Banjanovic-Mehmedovic ${ }^{1}$, Izet Jagodic ${ }^{2}$ \\ ${ }^{1}$ The Faculty of Electrical Engineering, University of Tuzla, Tuzla, Bosnia and Herzegovina. \\ 2 Faculty of Electrical Engineering, University of East Sarajevo, East Sarajevo, Bosnia and Herzegovina. \\ * Corresponding author. Tel.:++38761410669, email: suad.kasapovic@untz.ba \\ Manuscript submitted July 17, 2016; accepted October 16, 2016. \\ doi: 10.17706/jsw.11.11.1154-1161
}

\begin{abstract}
The aim of this paper is to demonstrate, how to communicate the vehicle between themselves in a heterogeneous vehicles network, and show on which way is done the exchange of information with the infrastructure, to overcome the shortcomings of using a single wireless technology. DSRC does not offer enough good coverage and range around intersections in urban areas for specific applications. On the other side, as an alternative to overcoming these deficiencies proposed LTE, advanced mobile communications technology. The evaluation of performance is usually done by means of simulations in particular the programming software, which integrates tools to support Wi-Fi, IEEE 802.11p, mobile technology and feedback mobility. For the realization of heterogeneous networks vehicle that has support for LTE is used open source simulator for communication between vehicles and infrastructure Veins LTE, composed of a network simulator Omnet++ and traffic simulator SUMO. These two simulators are working in parallel, and allow modeling of communication between vehicles.
\end{abstract}

Key words: Veins LTE, heterogeneous vehicular network, LTE, DSRC, IEEE 802.11p, Omnet++, SUMO.

\section{Introduction}

Do not change the font sizes or line spacing to squeeze more text into a limited number of pages. Use italics for emphasis; do not underline. By integrating technologies for information and communications, intelligent transportation systems (ITS) enable authorities, operators and individual travelers to make better in-formed and coordinated decisions. A precise description the weather or an early warning system on encountering danger, would be very useful for drivers. IEEE $802.11 \mathrm{p}$ is the standard that supports ITS applications in Vehicular Ad hoc NETworks (VANETs). Due to its limited radio range and without a pervasive roadside communication infrastructure, $802.11 \mathrm{p}$ can only offer intermittent and short-lived V2I connectivity. LTE and LTE-A are the main candidate access technologies which have different characteristics and can match the vehicular application's requirements, more or less effectively. Like all cellular systems, it can benet from a large coverage area, high penetration rate, and high-speed terminal support. In the regions completely covered by the mobile network, LTE can organize the vehicular network in a set of clusters, in a centralized manner. Therefore, it is necessary to demonstrate the impact VANET networks before the deployment, and to this end conducted computer simulations that include communication models, models of mobility vehicles and models VANET applications. The aim of this article is to show you how you can best explore the heterogeneous network technologies for vehicles to overcome the disadvantages when using a single wireless technology [1]. Performance evaluation is usually done by means of simulation, which requires integrated tools that support Wi-Fi, IEEE 802.11p, mobile technology and mobility feedback [2]. 
Most current network simulators for vehicles do not have support for heterogeneous networking, especially for LTE (Eng. Long Term Evolution (LTE)). It is therefore used new integrated simulation framework based on the popular Veins framework called Veins LTE [3].

Some recent publications have focused on the grouping of vehicles (create clusters) and heterogeneous networking in order to execute the distribution of messages and to reduce delays [4]. The very dynamic network topology due to vehicles high velocity, aiming to ensure acceptable V2V and V2I communications. Most of the solutions proposed for handling these issues are based on the creation of dynamic clusters to self-organize the IEEE 802.11p vehicular network. Clustering in VANET aims to organize vehicles into groups based on some specific common characteristics.

The need for heterogeneous networking appeared because of the technique when applied individually have drawbacks. Older vehicles are not equipped with the necessary modules WLAN technologies such as IEEE 802.11p, but there is a suspicion in the possibility of covering densely populated urban areas [5]. IEEE 802.11p is capable of handling active road safety when vehicles are sparsely distributed within the same area or with limited mobility support. Also, LTE based technology has insufficient capacity to support frequent changes messages in VANET network because it is intended for other types of services.

\section{The Simulation Tool - Veins LTE}

In order to simulate the mobility of vehicles, we used the traces that were formed before the simulation. Mobility simulator works in parallel with the network simulator that are directly connected. This work presens use of simulator called VeinsLTE. VeinsLTE combines the functions of Veins with SimuLTE for LTE support [6]. The main objective was to integrate LTE in the Veins system, to easily develop new applications. SimuLTE simulator allows simulation LTE and provides a detailed model of the complete LTE stack. Integration SimuLTE in Veins is a demanding work and SimuLTE requests initialization of all nodes at the beginning of the simulation itself. It is not useful for the simulation of vehicle networks as vehicles enter and leave covered scenario during the simulation. Therefore, it was necessary ability to add and remove vehicles on SimuLTE framework - from the NIC (eng. Network Interface Controller (NIC)) in the IP stack. As a result, the obtained a framework to simulate a heterogeneous network of vehicles, where users can deploy the application on top of the stack. After creating the application, start-up and configuration simulation to collect results is supported by Omnet++. Protocol layers of the simulated vehicle in a heterogeneous network are shown in Fig. 1. which includes LTE and IEEE 802.11p network interface [7].

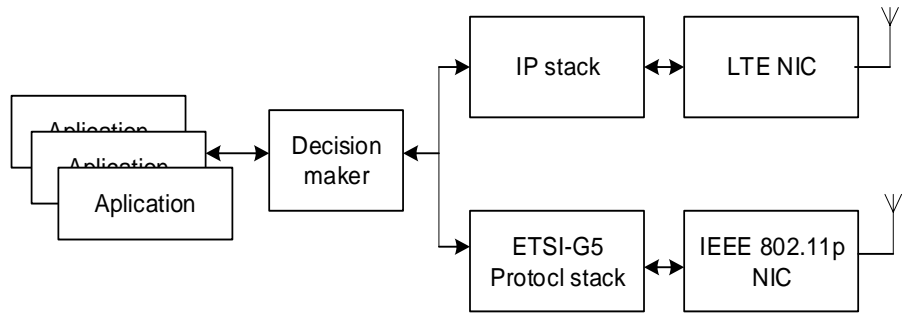

Fig. 1. Protocol layers of heterogeneous vehicular network.

At the top of the stack are the application layer and a layer of decision. The application should be able to either explicitly decided to use the network stack or let you decide make that decision. Under the decision-makers there are two flows, one for technology LTE and 802.11p [8]. Both have a special adaptation layer where additional information is added to the packets. These accessories can be flags and headers that are not needed in other network technologies. Under a layer adaptations, each stack has a MAC (engl. Medium Access Control (MAC) layer and layer NIC. Due to the nature of our stack, it is easy to develop 
new applications using this simulator [1]. With Omnet++ is easily modify various parameters of various layers and together with VeinsLTE comes with an advanced IDE which is based on Eclipse and useful GUI for running simulations. In Omnet ++ tools, simulation models are configured for execution using the configuration file with the .ini extension (INI files, Fig. 2.). The table below list shows the part of current settings section and all other sections of which received items. Fig. 2. shows the dimensions defined areas of simulation. The width and length are set to $20000 \mathrm{~m}$ with a height of $50 \mathrm{~m}$. Furthermore, manager module creates and runs the vehicle with the help of a submodule TraCIMobility and manager.updateInterval parameter specifies the time interval updates positions host and is set to 0.1 seconds.

\begin{tabular}{|l|l|}
\hline$*$ *.playgroundSizeX & $\triangle 20000 \mathrm{~m}$ \\
\hline *.playgroundSizeY & $\$ 20000 \mathrm{~m}$ \\
\hline *.playgroundSizeZ & $\$ 50 \mathrm{~m}$ \\
\hline *.annotations.draw & $\triangle$ false \\
\hline
\end{tabular}

Fig. 2. The file in the INI file editor.

Figure 3. shows the parameters assigned connectionManager modules and four parameters used to calculate the limits of interference distance between nodes such as pMax, sat, alpha and carrierFrequency. pMax is the maximum transmit power of the network and its value is set to $20 \mathrm{~mW}$, sat the minimum threshold of signal attenuation (eng. signal attenuation threshold) and its value is $-89 \mathrm{dBm}$, alpha parameter is the value of the coefficient of minimal path loss and carrierFrequency refers to the minimum frequency of the carrier channel and its value is $5.89 \mathrm{GHz}$.

\begin{tabular}{|c|c|}
\hline *.connectionManager.pMax & $\triangle 20 \mathrm{~mW}$ \\
\hline * *.connectionManager.sat & (4) -89dBm \\
\hline *.connectionManager.alpha & $\triangle 2.0$ \\
\hline * *.connectionManager.carrierFrequency & $45.890 \mathrm{e} 9 \mathrm{~Hz}$ \\
\hline *.connectionManager.sendDirect & $\Delta$ true \\
\hline *.**.nic80211p.mac1609_4.useServiceChannel & $\triangle$ false \\
\hline *.**.nic80211p.mac1609_4.txPower & $\triangle 20 \mathrm{~mW}$ \\
\hline *.**.nic80211p.mac1609_4.bitrate & A $18 \mathrm{Mbps}$ \\
\hline *.**.nic80211p.phy80211p.sensitivity & $4-89 \mathrm{dBm}$ \\
\hline *.**.nic80211p.phy80211p.maxTXPower & $\Delta 10 \mathrm{~mW}$ \\
\hline
\end{tabular}

Fig. 3. 802.11p specific parameters - NIC settings.

The NIC settings, data rate set to $18 \mathrm{Mbps}$ and sensitivity to $-89 \mathrm{dBm}$. After having made the final scenario and you have set all the parameters, you can run a simulation. The simulation runs in Tkenv graphical environment that supports execution, animation, tracing and debugging simulation. Tkenv is intended for presentation and educational purposes, as it allows the user to have a detailed picture of the state of the simulation at any time and to follow what is happening in the network. The most important opportunities Tkenv graphical environment are: animation flow messages, graphical display of statistics and output vectors during the execution of the simulation.

\section{The Simulation Scenario}

Algorithm networking vehicles, which is implemented in the simulator, in a heterogeneous network, uses an algorithm for collision avoidance, where vehicles using dedicated short-range communications (DSRC) to exchange their current position and form clusters vehicles [9]. Data on the cluster are transmitted via LTE to a central server, which is then able to alert the clusters in the case come near the vehicle. Figure 4 . shows communication with the server so that vehicles exchanged messages via LTE and 802.11p. After running the 
simulation, executed events according to a predetermined script. Scenario examples treated in this article contains 10 vehicles marked nodes 0-9 that 'throw' 'on the field simulation every 5 seconds and one vehicle in one of the directions. Vehicles are therefore appearing at the beginning of the route. All vehicles have the same route and the same speed. Except for collision avoidance, such data can be used to control intersections or route planning. Vehicles appear at the beginning of the route, and with Fig. 4. can be seen that there are two directions that are approaching the main intersection. During the simulation, the vehicle exchanged messages within their structure.

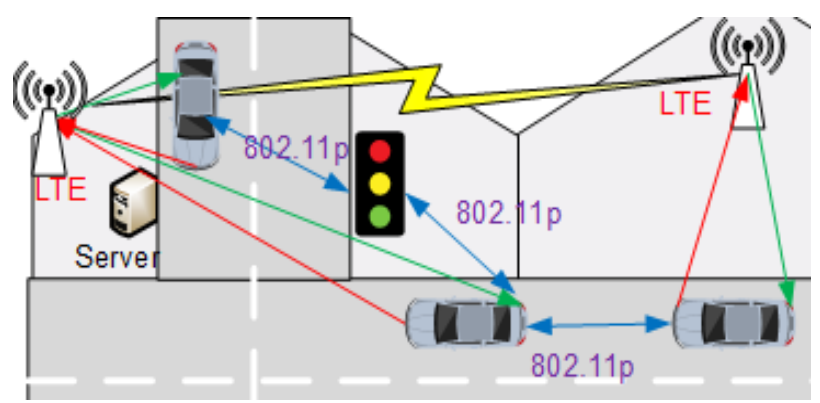

Fig. 4. Communication with the server.

The modules themselves send messages, so-called self-messages (abbreviated selfmsg). Nearly all the simulation models have a schedule of future events in order to implement the timer, delay, etc., and therefore used selfmsg messages. These messages are presented with cMessage class, which is defined message class in Omnet ++. cMessage objects can represent events, messages, work or other entity for the simulation. In the event that these are network packets, used cPacket subclass. Fig. 5. shows LteAirFrame packets received by the base station of the channel, between the the node with the identification srcID $=1025$ or 1 vehicle and a single base station and layout examples type selfmsg.

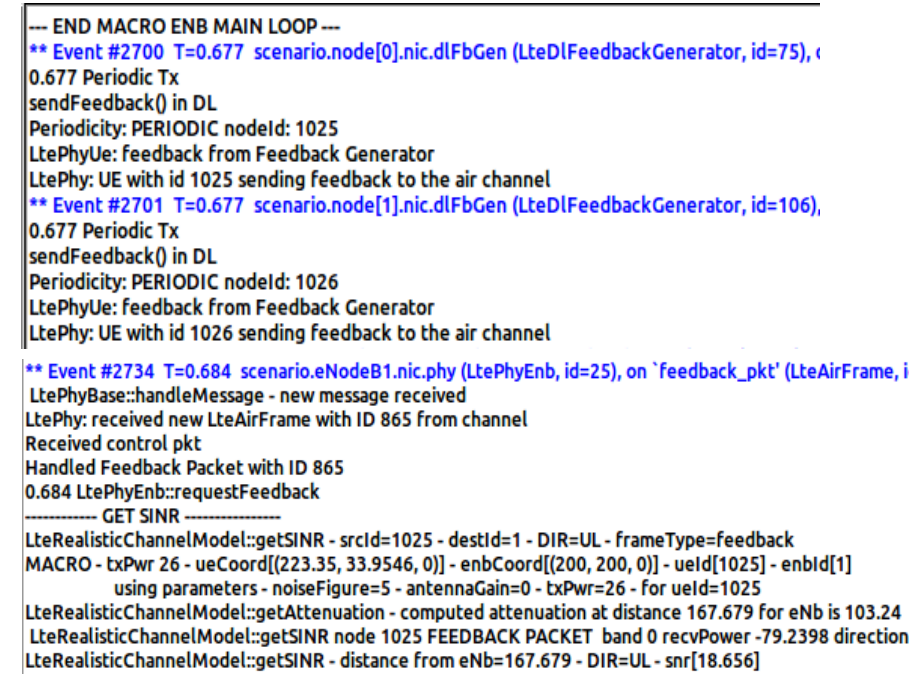

Fig. 5. Examples of selfmsg in Tkenv environment and content of LteAirFrame packet.

All vehicles, which is a notification message is intended, receiving first LTEAirFrame packet of channels. The modules, which listen for messages, can assess the noise or interference on the basis of information from the message. In the case of vehicle-to-infrastructure, in the context of LTEAirFrame have written information on the ratio of signal-to-interference plus noise for both uplink and downlink. Module Physical layer calculates interference on the basis of several parameters, some of which are antenna gain, transmit 
power, noise figure, and weakening others. Fig. 6. shows an example of animation sending LteAirFrame packet.

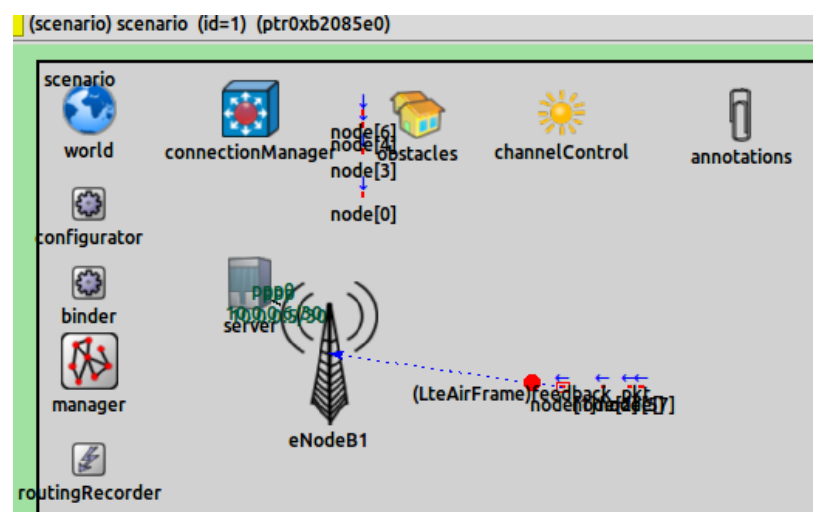

Fig. 6. Animation sending LteAirFrame packets.

\section{Results and Analysis}

The output vector files can store information about the behavior of simulations over time, while output scalar files contain summary statistics such as the number of packets transmitted, number of packets lost, the number of packets received, and so on. Using these two files simulation results can be visualized through Analysis Editor in the Omnet++ IDE and shows the number of sent and received messages LTE for each of the ten nodes individually. Since the DSRC by nature broadcast technique, ie. when a node sends a packet given node package that can be accepted by all other nodes in the simulation, so the simulator for this characteristic that captures information about how much the node received DSRC message. While the simulation is running, in addition to detailed data being printed in Tkenv environment, also is printed and data in the terminal, which are tied to send messages between nodes, but also to send messages from the node to the server. Each node in this scenario is identified by its assigned private IP address and its identification number, which corresponds to the number of nodes. In the same moment, node 5 sends a message server at the time of receipt of the message returns a reply message to a specific IP address that belongs to the node 5 . After sending the messages from the server, node 5 has reported that he has received the message from the server. The second part of the print results in the terminal, it can be seen that node 1 sends the message to the server which is in Fig. 7. marked with yellow. Upon termination or completion of the simulation, printed result, how much was received LTE messages on the server.

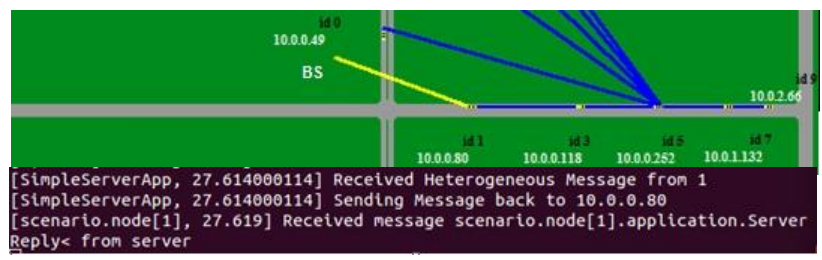

Fig. 7. Communication between vehicles and communication with the server.

Based on the time that elapses from the moment when one node sends a message to another node, and when the sender node receives the response, it is possible to create a diagram of the response time for communication between vehicles. With the analysis of the results will decide who makes the decision about whether the individual applications to send messages via LTE or using IEEE 802.11p, can be analyzed and other parameters, such as throughput, utilization, received broadcast packets, number of lost packets, the number of sent and received packets with lower or higher layer. In addition to the LTE received messages, in 
this analysis can be seen and the number of received DSRC messages for each node. Fig. 8. shows the exchange DSRC messages between the vehicles marked with blue, and in a way that node 5 sends a message exclusively node 9 , but all other nodes participating in the simulation also received a message from node 5 .

Fig. 9. shows the ratio of the number of packets lost on route from sender to recipient, and the number of packets lost due to signal interference plus noise, as well as the total number of lost packets. Diagram shows the ratio of the total number of packets received and the number of lost packets for each node individually during the simulation. Fig. 10. shows a graph of busy time for each node in seconds. Busy time is defined as the time at which the particular vehicle involved in the communication vehicle-to-vehicle or vehicle-to-infrastructure communication. If the value of the occupancy is higher, it means that the vehicle is more engaged in the communication, either with other vehicles or with radio statio unit. Busy time depends on the number of vehicles that communicate on the way to the destination. If the communication between the vehicle radio station units (RSU) or not busy time of the vehicle is considered to be larger relative to the other nodes. Heterogeneous networks have more time, which can be used to send other security messages between vehicles and the radio station units.

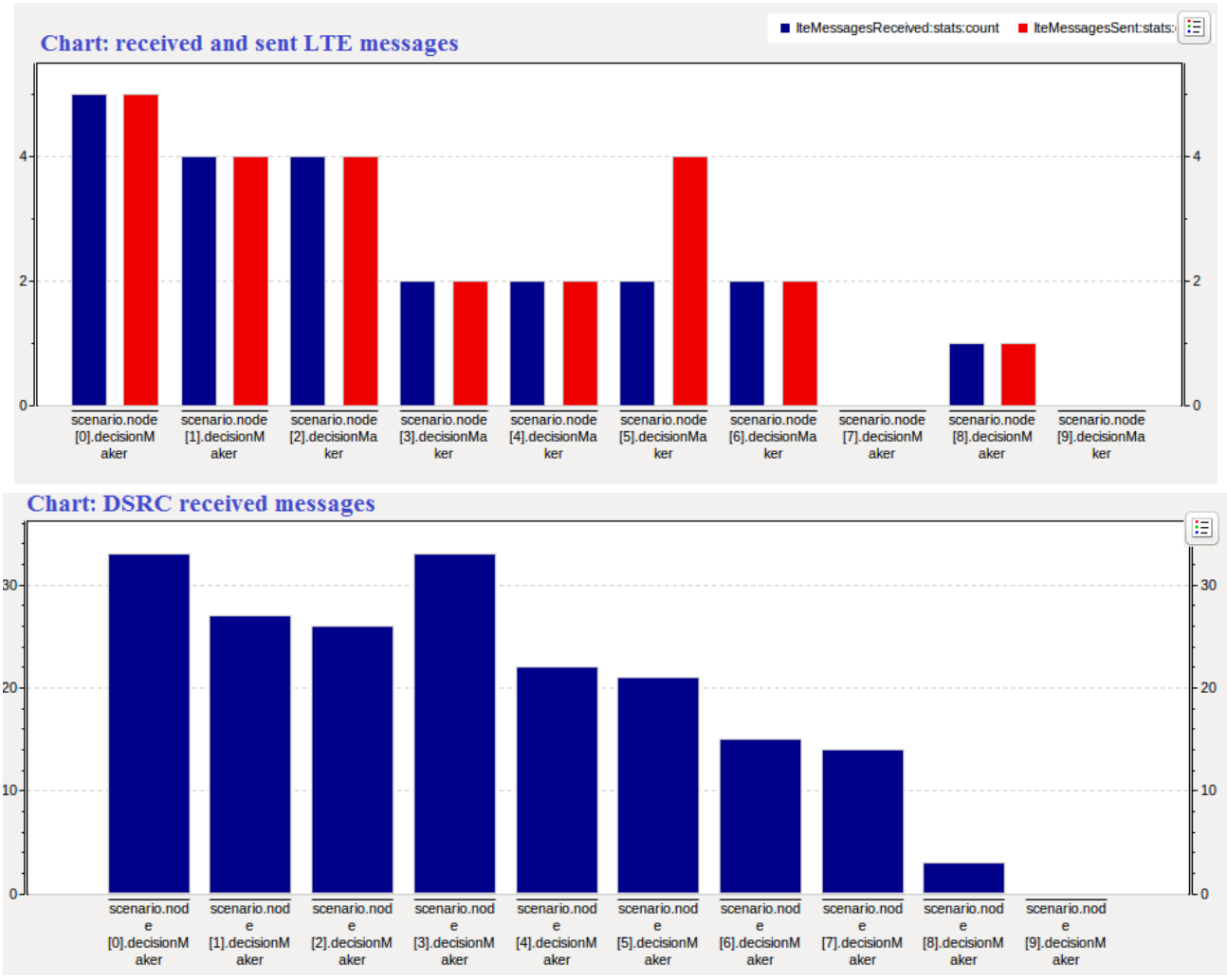

Fig. 8. Number of sent and received LTE and DSRC messages for each nod.

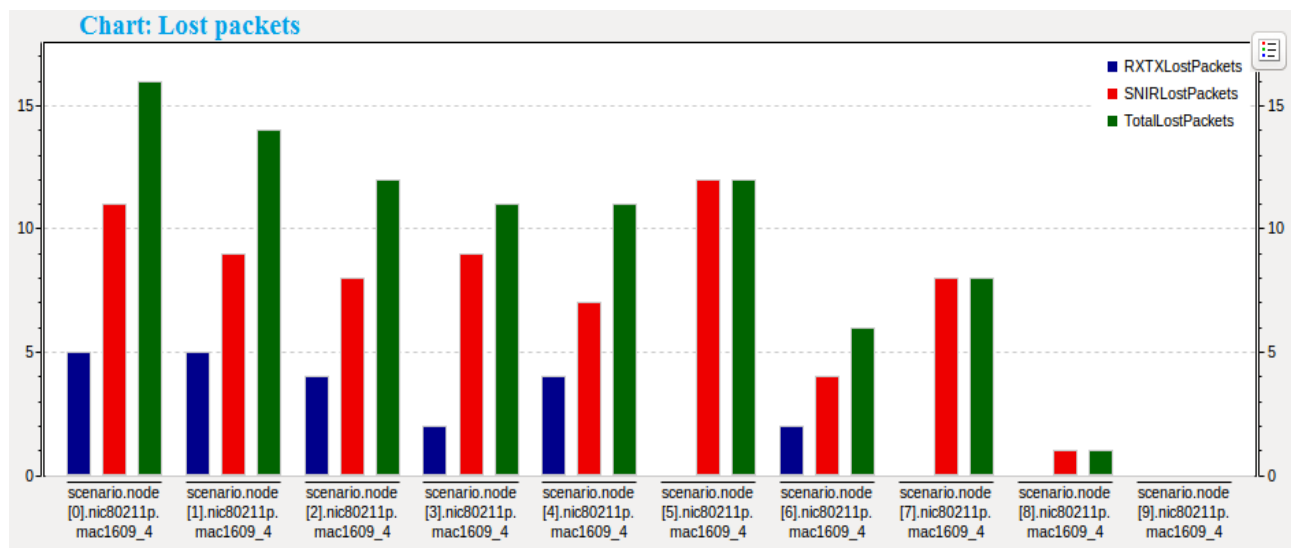




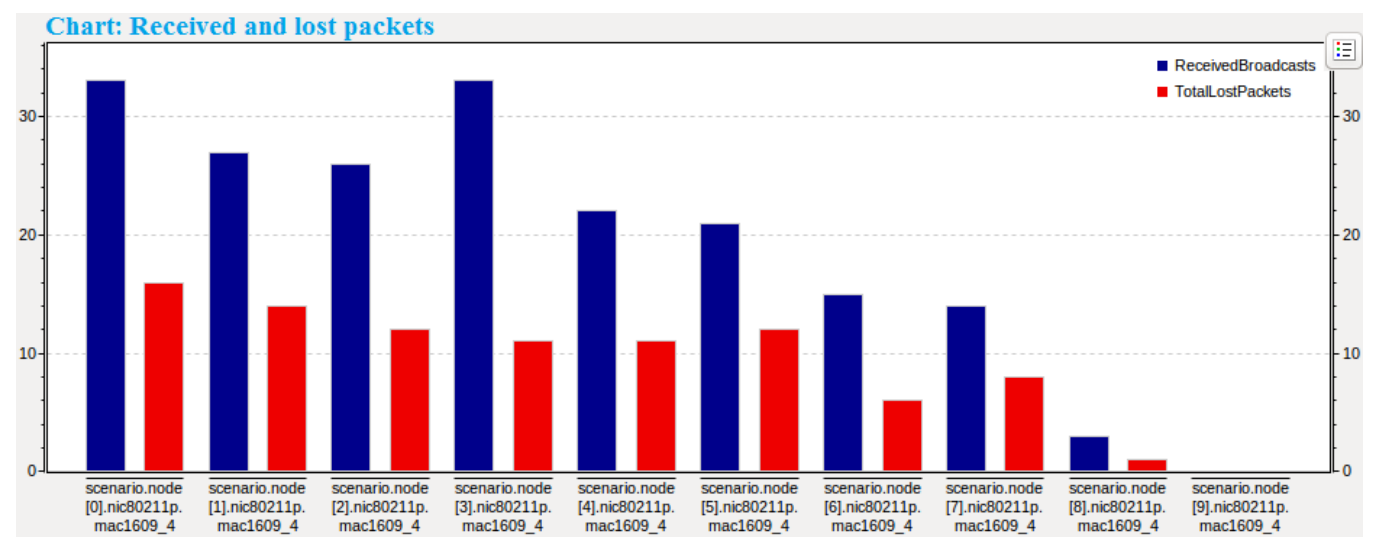

Fig. 9. Lost pasckets and comparation received and lost packets.

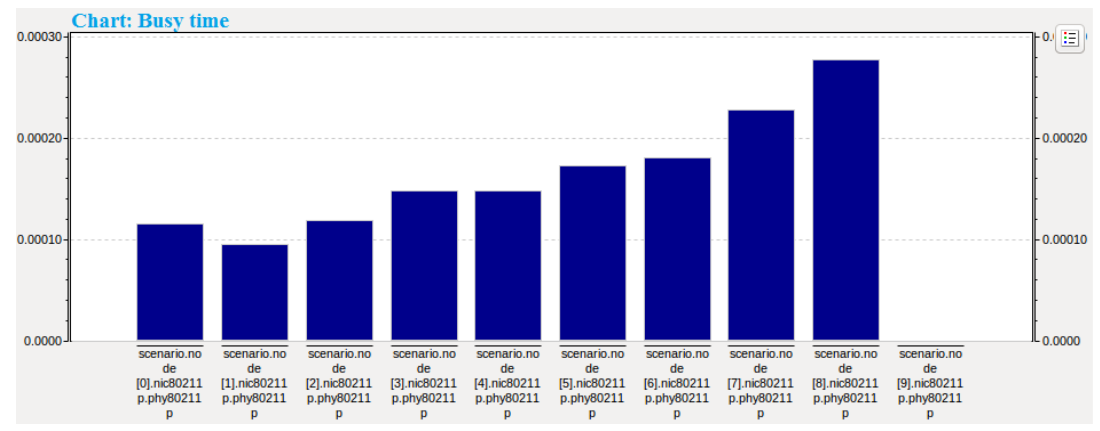

Fig. 10. Busy time.

\section{Conclusion}

This paper presents a simulator that can be used in a heterogeneous network of vehicles, using wireless technology 802.11p and LTE. In the present scenario analyzed is network of vehicles that mutually exchanging messages via the IEEE 802.11p and LTE. What is important note, that LTE offers higher network capacity and greater support mobility in relation to the IEEE $802.11 \mathrm{p}$ standard. Sharing of data within heterogeneous networks vehicles often plays an important role in traffic safety. Such information must be true and accurate, but also must be available at any given moment, without much delay and the evident loss of data. The use of heterogeneous networks of vehicles, in many ways they can prevent chain collisions, unnecessary waiting in lines for work on the road, and many other unpleasant situations, but also can provide very stringent safety requirements that would make driving safer. The results obtained by simulation heterogeneous networks have showed considerable performance improvements over the use of single access technology for the vehicular networking, which consequently further justifies the need of comprehensive study into the feasibility of one over the other.

\section{References}

[1] Florian, H., \& Falko, D. (2014). Christoph sommer: A simulator for heterogeneous vehicular networks. Proceedings of the 6th IEEE Vehicular Networking Conference (pp. 185-186).

[2] Zeeshan, H. (2014). "Fethi filali: LTE and IEEE 802.11p for vehicular networking: A performance evaluation. EURASIP Journal on Wireless Communications and Networking.

[3] Veins Simulator. Retrieve from http://veins.car2x.org/

[4] Agon, M., Florian, H., \& Falko, D. (2015). Christoph sommer: Cluster-based transmit power control in heterogeneous vehicular networks. Proceedings of the 7th IEEE Vehicular Networking Conference Kyoto. 
[5] Noori, H., \& Valkama, M. (2013). Impact of VANET-based V2X communication using IEEE 802.11p on reducing vehicles traveling time in realistic large scale urban area. Proceedings of the 2nd International Conference on Connected Vehicles \& Expo.

[6] Christoph, S., \& Falko, D. (2014). Vehicular Networking. Cambridge University Press.

[7] Florian, H. (2015). Heterogeneous vehicular networking. Proceedings of the International Conference on Networked Systems.

[8] Florian, H. (2015). Approaches to heterogeneous vehicular networks. Proceedings of the of 3rd GI/ITG KuVS Fachgespräch Inter-Vehicle Communication.

[9] Lung-Chih, T., Jorge, M., Mario, G. (2013). Christoph sommer: A cluster based architecture for intersection collision avoidance using heterogeneous networks. Proceedings of the 12th IFIP/IEEE Annual Mediterranean Ad Hoc Networking Workshop.

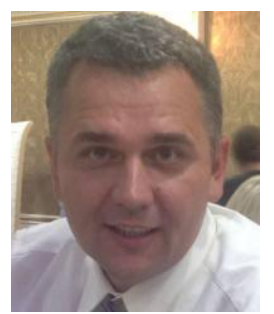

Suad Kasapovic graduated from the Faculty of Electrical Engineering in Tuzla in 1996. He received his scientific degree of master of science from the Faculty of Electrical Engineering and Computing, University of Zagreb 2002, and the degree of doctor of science in electrical engineering, the fields telecommunications and informatics, 2007 . He works at the University of Tuzla as an associate professor in the field of Telecommunications. Area of interest are wireless networks, design of IT computer systems \& networks and software-defined networks.

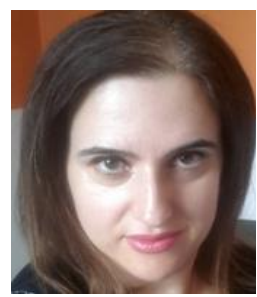

Samra Mujacic is currently working as an associate professor at Faculty of Electrical Engineering, University of Tuzla, Bosnia and Herzegovina. She received her Ph.D., M.S. and B.S. degree in electrical engineering from Faculty of Electrical Engineering and Computing, University of Zagreb, Croatia. Her research interests include multimedia systems, communications and services, interactive and adaptive hypermedia and e-learning.

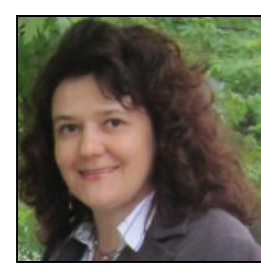

Lejla Banjanovic-Mehmedovic received the B.S. degree in 1989 and the M.S. degree in 1999 in electrical engineering both from the University of Sarajevo, Bosnia and Herzegovina. She received her Ph.D. degree in electrical engineering in 2006, from University of Zagreb, Croatia. Her current research interests include intelligent system's control, mobile robotics, distributed real-time systems and intelligent transport systems. Prof. Banjanovic-Mehmedovic is senior member of IEEE.

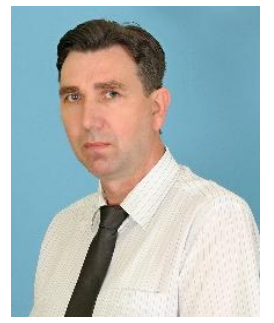

Izet Jagodic obtained his degree in electrical and electronics engineering in 1990 from Faculty of Electrical Engineering the University of Sarajevo, Bosnia and Herzegovina and a master's degree in telecommunications in 2011 on the University of East Sarajevo, Bosnia and Herzegovina. His research interests include computer networks, wireless sensor networks, advanced telecommunication systems. 